\title{
Factores organizacionales en una institución educativa privada del Callao-Perú
}

\section{Kamachinakuykuna kayninkuna huk nunap yaćhachina yaćhachinawasićhu Kallawkaćhu}

\author{
Kantagantsipage patoitagantsikë kara \\ ogomentotsikë kasintagantsi kara kallaokë
}

Recibido: 11 febrero 2020 Aprobado: 22 abril 2020

Rosa Milagro de Maria Delgado Herrera

Nacionalidad: Peruana / Filiación: Pontificia Universidad Católica del Perú Correo: rosa.delgadoh@pucp.edu.pe / ORCID: https://orcid.org/oooo-0002-9024-875X

Alex Oswaldo Sánchez Huarcaya

Nacionalidad: Peruana / Filiación: Pontificia Universidad Católica del Perú Correo: aosanchezh@pucp.edu.pe / ORCID: https://orcid.org/oooo-0003-3902-5902

\section{Resumen}

El presente artículo tiene como objetivo analizar los factores organizacionales que, desde la gestión educativa, garantizan la eficacia escolar en una institución privada del Callao. A través de la revisión de la bibliografía se contextualiza y define a la eficacia escolar, se recogen sus características y se identifican los factores organizacionales propios de esta. Asimismo, se definieron los temas preliminares. En base a estas categorías se estableció la guía de entrevista semiestructurada la cual fue aplicada a docentes de la institución previamente descrita. La información recogida y organizada como producto de la revisión bibliográfica fue contrastada con la información resultante de las entrevistas aplicadas. Fruto de la discusión e interpretación de los resultados se identificaron nueve factores organizacionales presentes en la institución analizada. Al final de la investigación se concluye que existe presencia de factores organizacionales en la institución, además se asume que la eficacia escolar no se logra siguiendo una fórmula o lista de cotejo, pues su logro depende de variables propias de cada escuela.

\section{Palabras clave:}

Factores organizacionales, eficacia escolar, modelo de gestión educativa estratégica.

\section{Lisichiku limaykuna:}

Kamachinakuykuna

kayninkuna, yaćhana atipay, allip yaćhay lulanalikanapa.

\section{Nibarintsipage katingatsaro:} kantagantsipage patoitagantsikë, kaninaro kara ogotagantsikë,angankero kaninansti ogenganepage. 


\title{
Organizational Factors in a Private School of Callao
}

\begin{abstract}
This article aims to analyze the organizational factors that, from the educational management, guarantee school effectiveness in a private institution of Callao. Through the review of the bibliography school effectiveness is contextualized and defined, its characteristics are collected and its organizational factors are identified. Likewise, preliminary issues were defined. Based on these categories, the semi-structured interview guide was established, which was applied to teachers of the previously described institution. The information collected and organized as a product of the bibliographic review was contrasted with the resulting information from the interviews applied. As a result of the discussion and interpretation of the results, nine organizational factors were identified in the analyzed institution. At the end of the investigation it is concluded that there is presence of organizational factors in the institution, it is also assumed that school effectiveness cannot be achieved following a formula or checklist, since its achievement depends on the variables of each school.
\end{abstract}

\author{
Keywords \\ Organizational \\ factors, school \\ effectiveness, \\ strategic \\ educational \\ management \\ model.
}

\section{Fatores organizacionais em uma instituição educativa particular do Callao-Perú}

\section{Resumo}

Este artigo tem como objetivo analisar os fatores organizacionais que, a partir da gestão educativa, garantem a eficácia escolar em uma instituição privada do Callao. Através da revisão da bibliografia é contextualizada e definida a eficácia, suas características são coletadas e seus fatores organizacionais são identificados. Da mesma forma, foram definidos os temas preliminares. Com base nessas categorias, foi estabelecido o guia da entrevista semiestruturada aplicado aos professores da instituição descrita anteriormente. As informações coletadas e organizadas como produto da revisão bibliográfica foram contrastadas com as informações resultantes das entrevistas aplicadas. Em decorrência da discussão e interpretação dos resultados, foram identificados nove fatores organizacionais na instituição analisada. Ao final da investigação conclui-se que há presença de fatores organizacionais na instituição, atribui-se que a eficácia escolar não pode ser alcançada seguindo uma fórmula ou checklist, uma vez que sua realização depende das variáveis próprias de cada escola.
Palavras-chave:

Fatores

organizacionais, eficácia escolar, modelo estratégico de gestão educativa.

\section{Datos de los autores}

Alex Oswaldo Sánchez Huarcaya es docente e investigador en educación. Doctor en Filosofía y Ciencias de la Educación por la Universidad Humboldt de Berlín, Alemania. Magister en Gestión de la Educación por la Pontificia Universidad Católica del Perú. Miembro del Grupo de Investigación GEDEP - PUCP.

Rosa Milagro de Maria Delgado Herrera es docente e investigadora en educación y acreditación. Bachiller en Educación en la especialidad de educación primaria. Estudiante de la maestría de educación con mención en Gestión de la Educación por la Pontificia Universidad Católica del Perú. Miembro del Grupo de Investigación GEDEP - PUCP. 


\section{Comprendiendo la eficacia escolar}

"Si la varianza total del rendimiento no puede ser exclusivamente explicada por las diferencias entre los alumnos (v. gr. aptitudes, motivación, etc.), existen razones para pensar en la posibilidad de mejorar el rendimiento en las escuelas." (Klitgaard y Hall, 1974 citado en Báez, 1994)

A raíz del informe Coleman (1966) citado en Murillo et al (2000), el cual planteaba como uno de sus resultados más sorprendentes que la escuela ejerce una escasa influencia en el rendimiento escolar de los estudiantes, surgió un interés social por descubrir cuál es el impacto que tiene la escuela en los aprendizajes y cuáles son los factores escolares que los suscitan. Es así que surgen movimientos, investigaciones y líneas de trabajo cuyo objetivo ha sido estudiar la eficacia escolar.

Al hablar de eficacia escolar hacemos referencia a, tal y como mencionan Morley y Rassool (1999) "una mezcla de diferentes ideologías que incorpora conocimientos bajo el énfasis de la calidad educativa"(Citado en Blanco et al, 2008, p. 23). Pero ¿qué es la eficacia escolar? Sammons (2011) citado en Carreras (2013), Cueto, Ramírez \& León (2003) y Blanco et al (2008) coinciden en que se comprende por eficacia escolar a la capacidad que tienen las instituciones escolares de superar los aprendizajes esperados de los estudiantes -los cuales, tal y como mencionan Granvik et al. (2018:466), son definidos muchas veces "en base a su rendimiento inicial y a su historial social, económico y cultural"- y de asegurar su desarrollo a nivel integral -no solo a nivel del cumplimiento de metas y de la significatividad de los aprendizajes; sino también, tal y como mencionan Huamán (2018), Murillo (2004) y Cornejo \& Redondo (2007), del desarrollo de su autoconcepto, de su capacidad reflexiva y crítica, de su formación en valores, de su bienestar y satisfacción-. Esta concepción centrada en el estudiante como el centro del proceso educativo es complementada por Schereens (2004 citado en Huamán, 2018, p. 16), quien hace referencia a otro aspecto que es comprendido dentro de la eficacia escolar: "la efectividad de mejora de las condiciones en el ámbito escolar", las cuales involucran al maestro, al aula y las relaciones con la comunidad educativa.

Finalmente, el concepto de escuelas eficaces evoluciona y a partir de Carreras (2013) comenta que desde los años setenta las escuelas eficaces surgen como un movimiento alternativo que se centra en los procesos formativos que se generan al interior de la comunidad educativa en lugar de centrarse en el tradicional modelo "input-output". Como consecuencia el concepto implica la calidad educativa, pasando de centrarse en la obtención de resultados cuantificables a ser relacionada- tal y como mencionan Murillo \& Román (2007); Blanco et al (2008); Muñoz y Murillo (2010 citado en Huamán, 2018)- con conceptos como equidad, eficiencia y compromiso de los agentes involucrados en la labor educativa; siendo el objetivo principal la búsqueda constante de la mejora escolar.

\section{Las características de las escuelas eficaces}

Los siguientes autores Hofman, Hofman \& Gray (2015); Stoll \& Dean (1999); Hopkins, Ainscow y West (1994 citados en Murillo et al, 2000); Fernández (2004) coinciden en el siguiente planteamiento: sin prestarle atención a la cultura escolar es difícil hablar de mejoras escolares; ello debido a que la cultura es una característica indesligable de la escuela.

Murillo et al (2000) proponen que las escuelas eficaces poseen las siguientes características: compromiso y participación colectiva de la comunidad escolar -característica también 
mencionada por Cornejo \& Redondo (2007)-, implicación activa y positiva del profesorado y de los directivos en el proceso de aprendizaje (Gamazo et al., 2018; Purkey y Smith, 1983 citados en Báez, 1994)-, trabajo docente colaborativo, esfuerzo constante por el alcance de la calidad (de manera sistemática y organizada), adaptación del proceso de enseñanza y aprendizaje y de los procesos organizativos y relacionales, auto-motivación institucional por la mejora escolar, evaluación y acompañamiento constante del progreso de los alumnos (Davis \& Thomas, 1992; Purkey y Smith, 1983 citados en Báez, 1994; Wang, Walters \& Thum, 2012 citados en Granvik et al., 2018) y de los procesos.

Otras características, facilitación de contextos de aprendizajes (Huamán, 2018); las relaciones positivas y fluidas entre maestros y familias y la promoción de un clima institucional positivo/estimulante (Fernández, 2004; Cornejo \& Redondo, 2007; Stoll \& Dean, 1999; Davis \& Thomas, 1992), también tenemos, la adaptación curricular, la responsabilidad del centro sobre los recursos y la promoción del desarrollo de la autonomía (Gamazo et al., 2018).

Cornejo \& Redondo (2007) comparten las siguientes características: metas compartidas claras y definidas -también mencionado por Stoll \& Dean (1999), Davis \& Thomas (1992) y Fernández (2004)-, liderazgo educativo y pedagógico activo -planteado por Davis \& Thomas (1992); Gamazo et al (2018); Modin, Låftman \& Östberg (2017 citados en Granvik et al, 2018); Wang, Walters \& Thum (2012 citados en Granvik et al., 2018)-, focalización en los aprendizajes integrales -definido por Stoll \& Dean (1999)-, sentido de pertenencia, capacidad de aprendizaje de la escuela y desarrollo profesional -establecido por Gamazo et al (2018).

Davis \& Thomas (1992) consideran como características tener: grandes expectativas docentes sobre el rendimiento del estudiante -coinciden con Modin, Låftman \& Östberg (2017 citados en Granvik et al., 2018); Purkey y Smith (1983 citados en Báez, 1994)-, un entorno seguro y ordenado para el aprendizaje (Wang, Walters \& Thum, 2012 citados en Granvik et al., 2018), énfasis en el aprendizaje de habilidades básicas (Stoll \& Dean, 1999), mejora en el desempeño docente (Wang, Walters \& Thum, 2012 citados en Granvik et al., 2018), principios sobre gestión del aula (Gamazo et al., 2018).

Finalmente, se encuentra el tener una estructura escolar que promueva el aprendizaje (Modin, Låftman \& Östberg, 2017 citados en Granvik et al., 2018) y la participación de los padres de familia, el uso eficiente del tiempo en el aula y la flexibilidad, creatividad y adaptabilidad de las actividades educativas (Purkey y Smith, 1983 citados en Báez, 1994).

Las características previamente mencionadas han sido planteadas por los diversos autores como elementos presentes en escuelas que se identifican como eficaces, estas características son de naturaleza estructural, de gestión, de compromiso, de procesos, de pensamiento, de formación y de oportunidades; lo anterior permite comprender que la labor a desarrollarse es compleja pues demanda un trabajo institucional sistémico.

\section{La gestión educativa orientada hacia la eficacia escolar}

"Elementos claves para la mejora de las escuelas: en primer lugar, una administración que confíe en sus docentes y que apoye su trabajo. (...) una escuela en la que prevalezca la cultura del aprendizaje de todos (sólo se puede conseguir que los estudiantes aprendan si los docen- 
tes aprenden también), (...) y unos docentes comprometidos con sus estudiantes, su escuela y la sociedad." (Murillo \& Martínez, 2016:130).

Las investigaciones sobre la eficacia escolar han logrado demostrar que, contrario a los resultados del informe Coleman (1966, citado en Murillo et al, 2000), la escuela sí tiene influencia en los aprendizajes de sus estudiantes. En base a esta premisa, en este apartado se identificarán -desde la literatura- cuáles son los elementos que facilitan el logro de la eficacia escolar.

Blanco et al (2008), Edmonds (1982, citado en Báez, 1994), Instituto Internacional de Planeamiento de la Educación (2000), Muñoz et al. (2004, citado en Blanco, 2008), Fernández (2003 citado en Blanco, 2008), Blanco (2009), Sammons (2011 citado en Murillo \& Martínez, 2016), López \& Gonzales (2011 citados en Carreras, 2013), Murillo (2008), Hofman, Hofman \& Gray (2015), La Torre \& Filp (1990 citados en Huamán, 2018) plantean los siguientes factores como propios de las escuelas eficaces:

1. Sentido de comunidad: Comprendida como la existencia de metas claras, conocidas y compartidas por la comunidad escolar, y como el índice de grupalidad alto (trabajo docente colaborativo).

2. Liderazgo educativo y pedagógico: Centrado en la atención a la calidad del proceso de enseñanza-aprendizaje. La experiencia y el grado de actualización pedagógica del director son importantes para desarrollar este liderazgo.

3. Clima escolar y de aula: Entendido como ambiente ordenado y seguro que facilita y estimula el proceso de enseñanza-aprendizaje, las relaciones positivas entre los miembros de la comunidad educativa y la presencia de un ambiente afectivo y que fomenta el aprendizaje.

4. Organización del aula: Disposición y articulación de las actividades en el aula, la organización física de la misma para facilitar el aprendizaje.

5. Altas expectativas: Altas expectativas docentes sobre las posibilidades de aprendizaje de los estudiantes, de los directivos sobre el desempeño docente y de los padres de familia sobre el centro educativo.

6. Calidad de la enseñanza y del currículo: Enseñanza centrada en el aprendizaje a través de estrategias individualizadas orientadas a la formación integral de los estudiantes y a la atención a la diversidad.

7. Seguimiento y evaluación en todos los niveles: Acompañamiento del progreso de los estudiantes, del profesorado y de la institución con el objetivo de mejora continua.

8. Aprendizaje organizativo/ Desarrollo profesional: Apertura hacia la formación continua, el aprendizaje continuo y la innovación progresiva.

9. Compromiso e implicación de la comunidad educativa: Participación de las familias en las diferentes actividades escolares y apoyo en las tareas educativas de extensión (responsabilidad compartida).

10. Recursos educativos e infraestructura: Recursos materiales y humanos orientados a la promoción de la mejora educativa.

11. Gestión del tiempo: Distribución efectiva del tiempo en el aula de clases.

12. Estabilidad docente: Experiencia y bienestar docente como requisito previo para el buen desempeño. 
13. Cultura escolar positiva: Respetar el input con que ingresan los estudiantes, las diversas realidades de donde vienen, los diversos estilos de aprendizaje existentes.

14. Políticas escolares contextualizadas: Comprendidas como los lineamientos que guían el quehacer educativo, tienen el poder de mejorar o dificultar la labor educativa diaria.

En base a los factores previamente mencionados y al modelo de gestión educativa, se buscará identificar los elementos que desde la gestión educativa garantizan la eficacia escolar.

“La gestión educativa estratégica es vista como un conjunto de procesos teórico-prácticos integrados horizontal y verticalmente dentro del sistema educativo, para cumplir los mandatos sociales. Es un saber de síntesis capaz de ligar conocimiento y acción, ética y eficacia, política y administración en procesos que tienden al mejoramiento continuo de las prácticas educativas; a la exploración y explotación de todas las posibilidades; y a la innovación permanente como proceso sistemático." (IIPE, 2000:16)

La Secretaría de Educación Pública (2010) establece 9 componentes que le dan soporte al modelo de gestión educativa estratégica: El liderazgo compartido, el trabajo colaborativo, la planeación estratégica, prácticas docentes flexibles, la participación social responsable, la evaluación para la mejora continua, flexibilidad en las prácticas y relaciones, transparencia y rendición de cuentas, autonomía responsable/libertad en la toma de decisiones y corresponsabilidad/participación social responsable, características básicas y relación entre los componentes de la gestión educativa.

A luz de estos componentes se organizarán los factores organizacionales que desde la gestión educativa garantizan la eficacia escolar:

Tabla 1

Los factores organizacionales en el MGEE

\begin{tabular}{|c|c|c|}
\hline Componentes del MGEE & $\begin{array}{c}\text { Factores organizacionales de } \\
\text { la eficacia escolar }\end{array}$ & $\begin{array}{c}\text { Características de las escuelas } \\
\text { eficaces }\end{array}$ \\
\hline Liderazgo compartido & $\begin{array}{l}\text { Liderazgo educativo y } \\
\text { pedagógico }\end{array}$ & $\begin{array}{l}\text { Metas compartidas claras y definidas } \\
\text { Liderazgo educativo y pedagógico } \\
\text { activo }\end{array}$ \\
\hline Trabajo colaborativo & Sentido de comunidad & $\begin{array}{l}\text { Compromiso } \\
\text { Participación colectiva de la } \\
\text { comunidad escolar } \\
\text { Sentido de pertenencia } \\
\text { Participación de los padres de familia }\end{array}$ \\
\hline $\begin{array}{c}\text { Planeación estratégica y } \\
\text { participativa }\end{array}$ & & $\begin{array}{l}\text { Implicación activa y positiva del } \\
\text { profesorado y de los directivos en el }\end{array}$ \\
\hline $\begin{array}{l}\text { Participación social } \\
\text { responsable }\end{array}$ & $\begin{array}{l}\text { Compromiso e implicación de } \\
\text { la comunidad educativa }\end{array}$ & $\begin{array}{l}\text { proceso de aprendizaje } \\
\text { Auto-motivación institucional por la } \\
\text { mejora escolar }\end{array}$ \\
\hline \multirow[b]{2}{*}{$\begin{array}{l}\text { Evaluación para la mejora } \\
\text { continua }\end{array}$} & $\begin{array}{c}\text { Seguimiento y evaluación en } \\
\text { todos los niveles }\end{array}$ & $\begin{array}{l}\text { Evaluación y } \text { acompañamiento } \\
\text { constante del progreso de los alumnos }\end{array}$ \\
\hline & $\begin{array}{l}\text { Aprendizaje organizativo/ } \\
\text { Desarrollo profesional }\end{array}$ & $\begin{array}{l}\text { Capacidad de aprendizaje de la escuela } \\
\text { Desarrollo profesional } \\
\text { Mejora en el desempeño docente }\end{array}$ \\
\hline
\end{tabular}




\begin{tabular}{|c|c|c|}
\hline \multirow{4}{*}{$\begin{array}{l}\text { Flexibilidad en las prácticas } \\
\text { y relaciones }\end{array}$} & Organización del aula & $\begin{array}{l}\text { Entorno seguro y ordenado para el } \\
\text { aprendizaje } \\
\text { Principios sobre gestión del aula }\end{array}$ \\
\hline & Altas expectativas & $\begin{array}{l}\text { Grandes expectativas docentes sobre } \\
\text { el rendimiento del estudiante }\end{array}$ \\
\hline & Cultura escolar positiva & $\begin{array}{l}\text { Cultura escolar } \\
\text { Promoción del desarrollo de la } \\
\text { autonomía }\end{array}$ \\
\hline & Clima escolar y de aula & $\begin{array}{l}\text { Facilitación de contextos de } \\
\text { aprendizajes } \\
\text { Relaciones positivas y fluidas entre } \\
\text { maestros y familias } \\
\text { Clima institucional positivo/ } \\
\text { estimulante }\end{array}$ \\
\hline \multicolumn{3}{|l|}{$\begin{array}{c}\text { Transparencia y rendición } \\
\text { de cuentas }\end{array}$} \\
\hline \multirow{3}{*}{$\begin{array}{l}\text { Autonomía responsable } \\
\text { / Libertad en la toma de } \\
\text { decisiones }\end{array}$} & Gestión del tiempo & Uso eficiente del tiempo en el aula \\
\hline & $\begin{array}{l}\text { Recursos educativos e } \\
\text { infraestructura }\end{array}$ & $\begin{array}{l}\text { Responsabilidad del centro sobre los } \\
\text { recursos } \\
\text { Estructura escolar que promueva el } \\
\text { aprendizaje }\end{array}$ \\
\hline & $\begin{array}{l}\text { Políticas escolares } \\
\text { contextualizadas }\end{array}$ & $\begin{array}{l}\text { Trabajo docente colaborativo } \\
\text { Adaptación de los procesos } \\
\text { organizativos y relacionales }\end{array}$ \\
\hline \multirow[t]{2}{*}{$\begin{array}{c}\text { Corresponsabilidad / } \\
\text { Relación entre los } \\
\text { componentes de la gestión } \\
\text { educativa }\end{array}$} & $\begin{array}{l}\text { Calidad de la enseñanza y del } \\
\text { currículo }\end{array}$ & $\begin{array}{l}\text { Esfuerzo constante por el alcance de la } \\
\text { calidad } \\
\text { Adaptación del proceso de enseñanza } \\
\text { y aprendizaje } \\
\text { Adaptación curricular } \\
\text { Focalización en los aprendizajes } \\
\text { integrales } \\
\text { Énfasis en el aprendizaje de habilidades } \\
\text { básicas } \\
\begin{array}{l}\text { Flexibilidad, creatividad y y } \\
\text { adaptabilidad de las actividades } \\
\text { educativas }\end{array}\end{array}$ \\
\hline & Estabilidad docente & \\
\hline
\end{tabular}

Es importante comprender lo planteado por Murillo \& Martínez (2016), lo cual también es mencionado por Davis \& Thomas (1992) y Cornejo \& Redondo (2007), con respecto a la inviabilidad de establecer fórmulas para conseguir la eficacia en la escuela. Ello debido a que las escuelas no son instituciones aisladas, todo lo contrario, son instituciones cuyas características son producto del contexto en que se encuentran; es por ello que los factores que en algunas instituciones promueven la eficacia escolar, en otras no tiene el mismo impacto. Asimismo, el contexto no es el único elemento diferenciador entre escuelas, sino también su historia, su trayectoria, sus estructuras organizativas, su identidad y sus rutinas establecidas en base a sus experiencias. En base a ello se puede concluir que si bien analizar las escuelas eficaces e identificar los factores que tienen en común son dos pasos necesarios para la concientización y la mejora; es más importante conocer el cómo pasaron de ser simples características potenciales a definirse como factores de eficacia escolar. 


\section{Diseño metodológico}

La presente investigación pertenece a un enfoque cualitativo debido a que la metodología empleada busca comprender el complejo mundo de la experiencia vivida desde el punto de vista de las personas que la viven (Taylor y Bogdan, 1984). Experiencias en este caso relacionadas directamente a la labor educativa y a los elementos que en esta confluyen en su constante búsqueda de la excelencia educativa.

El estudio responde a la siguiente pregunta de investigación: ¿Cuáles son las percepciones docentes sobre los factores organizacionales que desde la gestión educativa garantizan la eficacia escolar en una institución privada del Callao? El objetivo general es analizar los factores organizacionales que desde la gestión educativa garantizan la eficacia escolar en una institución privada del Callao. Las categorías que orientan el recojo de las percepciones docentes, y sobre las que se basará la presente investigación son: evaluación para la mejora continua y flexibilidad docente, las cuales han sido planteadas por ser los componentes del MGEE que agrupan a los factores organizacionales con mayor presencia de eficacia escolar según los autores descritos en el marco conceptual.

La elección del centro educativo se basó en su pertenencia a un distrito representativo del Callao (Perú). Además, esta institución posee un alto porcentaje en los resultados de las pruebas PELA (Programa Estratégico Logros de Aprendizaje) y ECE (Evaluación Censal de Estudiantes) que se aplican anualmente a estudiantes de 2 do, 4 to y 6 to grado de primaria y 2 do de secundaria. Las pruebas responden a las áreas de comunicación, matemática y según el grado de ciencias. Estas pruebas son aplicadas por la DREC (Dirección Regional del Callao) y la UGEL (Unidad de Gestión Educativa local). Otro factor que se priorizó al momento de seleccionar la institución fue la accesibilidad y la disposición de los directivos para apoyar el desarrollo de la investigación.

Como se indicó previamente, el recojo de información se realizó por medio de una entrevista semiestructurada a cuatro profesoras del nivel primaria de la institución seleccionada. Las docentes han sido identificadas por el equipo directivo como las docentes más destacadas de la institución y que poseen un alto de nivel de aceptación por parte de los padres de familia.

Tabla 2

Caracterización de las entrevistadas

\begin{tabular}{|c|c|c|c|c|c|c|}
\hline $\begin{array}{c}\text { Código } \\
\text { empleados } \\
\text { en las } \\
\text { entrevistas }\end{array}$ & Sexo & Edad & $\begin{array}{c}\text { Nivel de } \\
\text { enseñanza }\end{array}$ & $\begin{array}{c}\text { Grado de } \\
\text { enseñanza }\end{array}$ & $\begin{array}{c}\text { Tiempo de } \\
\text { servicio }\end{array}$ & Cargo \\
\hline D1 & Femenino & 41 & $\begin{array}{c}\text { Educación } \\
\text { primaria }\end{array}$ & Tercer grado & 4 años & Docente de aula \\
\hline D2 & Femenino & 40 & $\begin{array}{c}\text { Educación } \\
\text { primaria }\end{array}$ & Segundo grado & 8 años & Docente de aula \\
\hline D3 & Femenino & 50 & $\begin{array}{c}\text { Educación } \\
\text { primaria }\end{array}$ & $\begin{array}{c}\text { Quinto grado- } \\
\text { Polidocencia }\end{array}$ & 19 años & $\begin{array}{c}\text { Docente de aula } \\
\text {-Coordinadora del } \\
\text { nivel }\end{array}$ \\
\hline D4 & Femenino & 47 & $\begin{array}{c}\text { Educación } \\
\text { primaria }\end{array}$ & Primer grado & 5 años & $\begin{array}{c}\text { Docente de aula } \\
\text { primario }\end{array}$ \\
\hline
\end{tabular}




\section{Intervención del estudio}

Como se mencionó anteriormente, se elaboró una entrevista semiestructurada como técnica de recojo de información en base a lo mencionado por Díaz-Bravo et al (2013), quien comparte que su uso es favorable en estudios descriptivos debido a su naturaleza flexible, adaptable, abierta, dialéctica e interpersonal. Esta entrevista tuvo como objetivo obtener las percepciones de las docentes consideradas personas claves (Dörner, 2012) sobre los factores organizacionales que desde la gestión educativa garantizan la eficacia escolar en la institución; para ello se siguió el siguiente proceso:

\section{Elaboración del guion de preguntas:}

Este estaba compuesto por 6 preguntas. Las cuatro primeras referentes a la primera categoría de estudio (Evaluación para la mejora continua) y las dos últimas referentes a la segunda categoría de estudio (flexibilidad docente).

\section{Validación del instrumento:}

El experto revisó las preguntas del instrumento verificando que estas respondan a las categorías seleccionadas, para ello hizo devoluciones en dos momentos, hasta que se validaron todas las preguntas que formaron parte de la entrevista.

\section{Aplicación del instrumento:}

Se coordinó con las informantes un horario y un lugar fuera de la institución debido a que ellas manifestaron que el tiempo dentro de esta estaba lleno de actividades. En base a las preguntas definidas en el guion de la entrevista se realizaron las seis preguntas predefinidas, se reformularon algunas preguntas en dos oportunidades para poder asegurar la comprensión de las mismas.

\section{Análisis de la información:}

A partir de las categorías de estudio definidas en base al marco conceptual realizado y empleadas en la elaboración del guion de preguntas seguido en la entrevista -previa información y firma del documento de consentimiento informado-, se procedió a la realización del análisis de lo recogido. Se elaboró una matriz de análisis en donde se realizó el vaciado de la información con el objetivo de codificar las transcripciones e identificar los elementos emergentes de las entrevistas realizadas, es decir, la información obtenida fue codificada (abierta) y procesada (Cuñat, 2007; Charmaz, 2007 citado en San Martín, 2014), de tal manera que surgieron los elementos emergentes que permiten el proceso de interpretación de los resultados.

La data recolectada se ha diferenciado y organizado en base a los resultados obtenidos como elementos emergentes y a sus categorías para su posterior análisis, siguiendo los principios éticos PUCP de compromiso y responsabilidad profesional, académica y científica y de Integridad y honestidad. Este proceso nos permite agrupar la información (figura 1) de manera que se pueden identificar los puntos consistentes que surgen y definen la naturaleza de los elementos que en la institución están actuando como facilitadores en el camino hacia la eficacia escolar. 
Figura 1:

Factores organizaciones que desde la gestión educativa garantizan la eficacia escolar en una I.E. del Callao a partir de las percepciones docentes

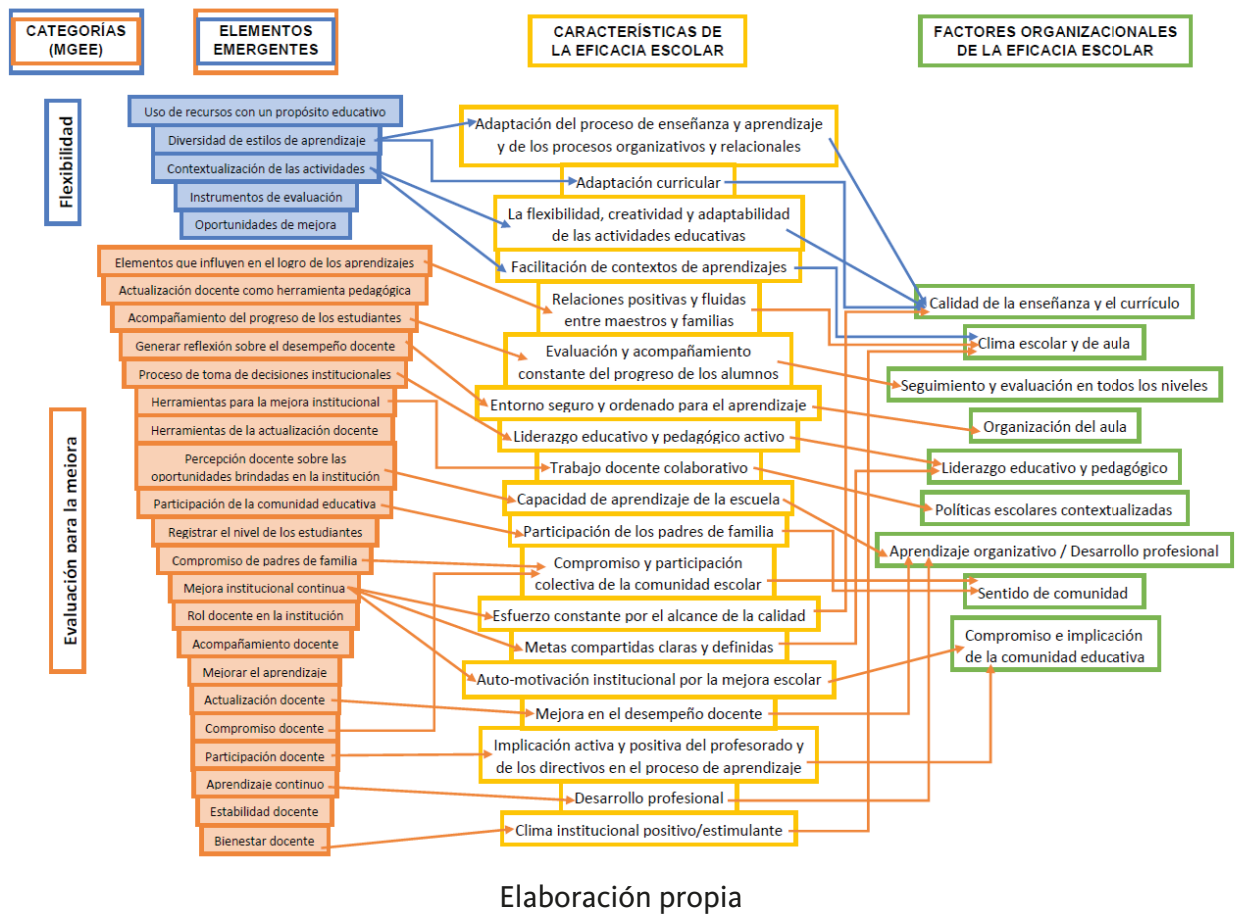

\section{Interpretación y discusión de hallazgos}

A partir de las preguntas establecidas hemos identificado veintiséis elementos, los cuales no solo hacen referencia a las categorías orientadoras, sino que dan información adicional que permite complementar el estudio. Asimismo, estos elementos emergentes los hemos relacionado a algunas de las características propias de la eficacia escolar y por ende a algunos de sus factores organizacionales correlativos.

Este análisis nos permite, además de conocer la realidad educativa, vislumbrar las potencialidades que tiene la institución en relación a la eficacia escolar y qué elementos deben desarrollarse de una mejor manera para poder alcanzar la calidad educativa esperada. Asimismo, este análisis está organizado a partir de dos categorías de estudio: evaluación para la mejora continua y flexibilidad docente, las cuales han orientado no solo la realización de las entrevistas sino también el proceso de interpretación y los resultados obtenidos.

El desarrollo de la categoría evaluación para la mejora continua comprende dos factores organizacionales de la eficacia escolar que han sido base en la elaboración de la entrevista: Seguimiento y evaluación en todos los niveles y Aprendizaje organizativo/ Desarrollo profesional. Sobre estos, se puede observar en las entrevistas que surgen veintiún elementos emergentes los cuales se relacionan a quince características que toda escuela eficaz posee. 
La evaluación para la mejora continua la comprendemos como una actividad que puede realizarse en tres niveles dentro de la institución: entre estudiantes y docentes -a nivel de aula-, entre los docentes y la institución, o en la misma institución. Sobre esto, en la entrevista se hace visible que el acompañamiento del proceso evaluativo de los docentes hacia sus estudiantes posee diferentes propósitos:

1. Registrar el nivel de los estudiantes, lo cual es mencionado por una docente, quien comenta que "los resultados de la evaluación a nivel de aula los empleo para poder registrar el nivel de mis estudiantes; (...) recojo información que me permite ver cómo van en cuanto a los objetivos de aprendizaje, si hay mejora o si no ha habido avance”. (D1_P1)

2. Acompañar el progreso de los estudiantes, lo cual es mencionado por una docente, quien comenta que el propósito del proceso evaluativo es el de "traer y desarrollar todos los cambios tecnológicos, renovar nuestro pensamiento, y respetando a la diversidad, acompañando constantemente al alumno". (D3_P5)

3. Mejorar el aprendizaje, lo cual es mencionado por una docente, quien comenta que "la idea es que todos aprendan y no se obstaculicen entre sí en el logro de las metas, porque si solo tengo en cuenta a un grupo es probable que no llegue a los otros y así promuevo diferencias y no las elimino". (D1_P5)

4. Generar reflexión sobre el desempeño docentes, lo cual es mencionado por una docente, quien comparte que "estar en el aula involucra repensar muchas veces las actividades planificadas en la marcha de la práctica, muchas veces lo que haces termina cambiando de lo que pensaste que ibas a hacer, lo único que sí se mantiene es el objetivo". (D4_P1)

El observar los cuatro propósitos evaluativos que surgieron como producto de la entrevista, surge un cuestionamiento importante ¿será que el no tener una visión compartida de la evaluación y acompañamiento que les realizamos a nuestros alumnos tiene un impacto en el alcance de la calidad educativa a nivel institucional? Ello nos permite tomar este punto como una oportunidad de mejora a nivel de institución a modo de cambio institucional.

En la entrevista, se hace visible también que el acompañamiento del progreso que hace la institución a sus docentes es percibido por estos como una realidad presente en la labor educativa, ello ha sido manifestado por la mayoría de las entrevistadas quienes afirmaron de manera consciente que este proceso se da no solo por parte de la dirección sino también de un especialista externo y que el propósito de este es hacer "que los resultados mejoren" (D4_P4). En el caso de la directora, "siempre está al pendiente de nuestra labor docente, ella nos habla desde su experiencia, nos aconseja y nos ayuda con los materiales para mejorar año tras año" (D1_P1). En el caso del especialista, "nos va asesorando para aplicar y mejorar nuestras estrategias, y viendo las competencias porque él también va evaluando a los alumnos y a nosotros para ver en qué nos puede ayudar" (D4_P4). Todo ello a través de "la revisión constante de nuestros documentos de planificación de clases" (D2_P4). Este acompañamiento docente es un elemento muy importante dentro de la labor educativa, pues no solo siente que es tomado en cuenta, sino que no ve a este proceso como un proceso supervisor sino de mejora, lo cual le permite comprender la viabilidad de este proceso y replicarlo en el aula con sus estudiantes.

Del mismo modo, en el análisis surgió la existencia de un propósito de mejora continua a nivel institucional, el cual fue identificado por las mismas docentes, quienes afirmaron que esta se da de dos maneras: A modo de “jornada de reflexión pedagógica o reuniones periódi- 
cas (...), donde se ven las problemáticas institucionales, en estas lo que se pretende es encausar y buscar alternativas que nos puedan ayudar a que nuestros estudiantes puedan mejorar (...), a ofrecer un mejor servicio, a mejorar nuestras prácticas docentes, a dar ideas que les permitan a los directivos responder a las demandas a las que se ven sometidos día a día." (D4_P2). Asimismo, mencionan que el proceso más profundo de mejora institucional "se da a fines de cada año, son evaluaciones que evalúan de manera general el servicio que brindamos. (...) Estas encuestas también tienen un espacio para sugerencias." (D3_P2).

Este proceso de mejora institucional podría ser tomado como un elemento que posee potencial a ser desarrollado, ello puesto que la frecuencia y la forma en que se desarrollan, según lo mencionado por las docentes, se centra en dar respuesta a problemáticas existentes y no da espacio a procesos de autorreflexión institucional. Para ello, cuenta con seis herramientas para la mejora institucional las cuales han sido identificadas por las docentes como: Trabajo docente colaborativo, comunicación constante, apertura institucional, trato dentro de la institución, respuestas a problemáticas inmediatas y capacitación previa a cambios; estas herramientas son elementos que tiene la institución y que le permitirán reforzar los elementos que aún requieren de trabajo para seguir en el camino hacia el logro de una escuela eficaz:

1. La participación docente, pues si bien es mencionado en la entrevista que los docentes se sienten tomados en cuenta y "parte de este cambio, porque algunas de las propuestas que surgen son por parte de los docentes, lo cual nos hace sentir escuchados." (D1_P6), se considera que esta participación no se debe limitar a ser parte del cambio sino también que deben tener la oportunidad de liderarlo. A pesar de ello, se puede decir que van un paso adelante, pues ya tienen definido el espacio solo falta organizarlo.

2. La definición del rol docente en la institución, este punto es muy importante pues se ha encontrado confusión en cuanto a la función del docente en este espacio. Algunas docentes comprenden que su labor se limita al trabajo y desarrollo de "los diversos tipos de inteligencia que tienen nuestros niños", a las "evaluaciones de manera constante", y a la realización de "talleres de refuerzo por las tardes" (D1_P3); mientras que otras se centran en la facilitación de un "ambiente tranquilo" de los estudiantes, y a centrarse "más en que desarrollen actitudes y aptitudes positivas que le permitan tener una buena experiencia en su estancia en el colegio." (D4_P5). Esto nos conflictúa y surge la interrogante: ¿será que el no tener una visión del rol que tiene el docente en la institución tiene un impacto en su desempeño y en el alcance de la calidad educativa a nivel institucional? Ello nos permite tomar este punto como una oportunidad de mejora a nivel de institución.

3. La estabilidad docente es otro elemento que debe ser tomado en cuenta, puesto que al verse relacionada a un proceso evaluativo que se da a fin de año - lo cual es mencionado por los docentes entrevistados quienes compartieron que "un elemento importante que tienen en cuenta en la renovación de contratos pues observan si es que ha habido mejoras a nivel profesional." (D2_P2)-, influye en el clima en que vive el docente todo el año limitando su desempeño docente a una mera respuesta a un estímulo externo.

Dentro de la categoría evaluación para la mejora continua se plantea como requisito la existencia de acciones, políticas y/o espacios que promuevan la formación y el aprendizaje continuo de los docentes. Sobre esto, las entrevistadas manifiestan que esta "es un proceso mediante el cual el docente se actualiza en sus conocimientos y procedimientos pedagógicos" (D2_P4) que tiene como propósito la mejora de la "propuesta educativa" ( $D_{3}$ P 4 ), orientada hacia "la búsqueda de innovar y mejorar a través del uso de nuevas estrategias, nuevas meto- 
dologías, nuevos materiales, el involucrar al niño en su aprendizaje, para que este juegue un rol principal" (D4_P3). Asimismo, manifestaron que si bien la institución brinda "capacitaciones a los docentes en los periodos donde no tenemos clases con niños" (D2_P2), estas se dan de manera "poco frecuente" (D2_P4) y que "si bien son útiles pues de una u otra manera me ayudan en mi trabajo a lo largo del año y contribuyen a mejorar mi enseñanza, tendrían un mayor impacto si fueran más frecuentes."(D4_P4). En este caso, se puede plantear a la actualización docente como una oportunidad de mejora que posee la institución muestra, puesto que la frecuencia con que se da en esta -según lo mencionado por las docentes- no asegura un proceso de aprendizaje continuo sistemático y por ende no asegura que tenga algún impacto en la labor educativa. Asimismo, esta oportunidad de mejora no solo hace referencia a la facilitación de cupos en talleres de actualización y capacitación docentes, sino que demanda -además de una política institucional- la definición de protocolos formativos que permitan el acompañamiento de la implementación y mejora de las oportunidades educativas que se dan en el aula (un retorno no solo en la mejora del equipo humano que labora en la institución sino también en la mejora del servicio brindado).

Si bien la actualización docente es un elemento muy importante en la labor educativa, no es la única manera en que se da el aprendizaje continuo, ello es sustentado por el $50 \%$ de las docentes quienes identifican que características como "el ser autodidacta, (...) el ser abierta a escuchar las recomendaciones de mis compañeros y a replicar las acciones pedagógicas exitosas de mis compañeros;(...) trabajar de manera conjunta con los padres de familia."(D2_P3) son elementos que también deben estar presentes en la labor docente, puesto que promueven el autoaprendizaje como otra manera de mejorar su desempeño en el aula.

Entre los elementos que influyen en el logro de los objetivos, las docente coincidieron en que: "Las relaciones de la familia, la situación económica, el nivel de formación de los padres de familia, la crisis de valores."(D3_P1), las “necesidades de apoyo socioemocional.”(D3_P5), “la motivación permanente, (...) el control de emociones, (...) la comprensión de valores”(D4_P5), "el input de los estudiantes, (...) la situación familiar"(D2_P6); tienen un alto nivel de influencia en el desempeño del niño en el aula y en el logro de sus aprendizajes. Ello se sustenta en lo mencionado por una docente quien asevera que "es importante al momento de trabajar con esta gran diversidad que traen los estudiantes, (...) contar con la ayuda de la institución y también la de los padres, pues son ellos los que nos dan el soporte y amplían nuestra labor." (D1_P6). Este punto es importante pues para alcanzar ser una escuela eficaz no solo se requiere del esfuerzo docente o de la escuela, sino también del compromiso de los padres, es decir, se necesita la participación de la comunidad educativa. Sin embargo, es necesario enfatizar que esto no se puede lograr si la institución no plantea espacios y protocolos de comunicación abierta entre todos los miembros de la comunidad escolar; este sería un elemento a tener en cuenta en un proyecto de mejora en la institución muestra.

El desarrollo de la categoría flexibilidad docente comprende tres factores organizacionales de la eficacia escolar que han sido base en la elaboración de la entrevista: Organización del aula, altas expectativas y una cultura escolar positiva. Sobre estos, se puede observar en las entrevistas que surgen cinco elementos emergentes los cuales se relacionan a cinco características que toda escuela eficaz posee.

La flexibilidad docente es definida por Ruiz y Parrilli (2015) como la capacidad que tienen los docentes de "ampliar y enriquecer la forma de aprender" de sus estudiantes. Esta flexibilidad es manejada por las entrevistadas dentro del aula en: 
1. La contextualización de las actividades que plantean, lo cual denota la flexibilidad, creatividad y adaptabilidad identificadas en el marco conceptual como características de las escuelas eficaces. Esta contextualización se da en torno a diferentes áreas según las entrevistadas, quienes manifiestan que al plantear sus sesiones deben "tener en cuenta la realidad del grupo para el que planifico las actividades."(D3_P5), deben tener en cuenta "las historias y cada una de las diversas culturas y prácticas diarias"(D1_P5), "la diversidad de mi aula, pues estas son de naturaleza emocional y/o cognitiva."(D2_P5), "el ritmo de aprendizaje de los estudiantes (...), los estilos de aprendizaje también varían, hay niños que son más metódicos, otros más prácticos, otros más reflexivos, y en todos hay que pensar. (...) Los tipos de aprendizaje que tienen los niños también deben ser considerados pues debo plantear actividades de diversa índole" (D4_P3). Esta contextualización, según lo mencionado por las entrevistadas, toma en cuenta la heterogeneidad presente en el aula, lo cual es un elemento presente en las escuelas eficaces.

2. La diversidad de estilos de aprendizaje, lo cual implica un proceso constante de adaptación curricular, del proceso de enseñanza y aprendizajes y de los procesos organizativos y relacionales identificados en el marco conceptual como características de las escuelas eficaces. Esta diversidad se da en torno a las necesidades que tienen las docentes en el aula, el $75 \%$ de las docentes manifestó poseer grupos de clase en los que los estilos de aprendizaje son "visuales, otros que son más kinestésicos, otros que son más auditivos, y así en sus diversas necesidades" (D3_P5). Asimismo, lo enunciado por las docentes no queda en mera identificación de la diversidad, pues plantean que organizan sus actividades en base a las necesidades presentes en su aula, "las actividades que planteo son de naturaleza diversa, pues tengo siempre que tratar de conectar con cada uno de ellos y que ninguno se sienta relegado." (D3_P5). Esta diversidad presente en el aula es tomada como punto de partida en la planificación y contextualización de las actividades a desarrollarse, lo cual es un elemento presente en las escuelas eficaces.

3. El uso de recursos con propósito educativo también ha sido un elemento emergente en las entrevistas realizadas, ello es mencionado por una docente quien manifiesta que en el caso de su aula "cuenta con un televisor y DVD (...) me ayuda a que se relajen (...) es muy útil pues lo niños de ahora tienden a ser más visuales(...); además, la selección de videos y música la hago con anterioridad para reforzar los temas de clase, así como los valores deseados" (D2_P5). Ello al ser analizados nos permite afirmar que dependiendo de la finalidad con que se usen los recursos del aula estos pueden contribuir a la labor docente o dificultarla. Es por ello que, si bien la misma docente planteó como una oportunidad de mejora el "contar con equipo multimedia para un mejor desarrollo de las clases, puesto que es más cercano a la realidad de los estudiantes" (D2_P5), podemos decir que si no se hace uso adecuado del recurso puede llegar a jugar en contra de la docente al perderse en la forma y no en el objetivo educativo.

4. Los instrumentos de evaluación, son importantes dentro de la labor educativa pues estos no solo denotan la flexibilidad docente sino también el objetivo de que este tiene para con sus estudiantes. La docente cuatro plantea que "constantemente evaluamos a los alumnos no solo con los exámenes semanales, mensuales y bimestrales; también nos basamos en lo que observamos de nuestros niños" (D4_P1). Al mencionar que no solo usa pruebas escritas en su evaluación, sino que también se basa en la observación individual de sus estudiantes es coherente en su afirmación de contextualización de las actividades y en la de su conocimiento de la diversidad de los estilos de aprendizaje. 


\section{Conclusiones}

Lo expuesto a lo largo de la presente investigación nos permite arribar a las siguientes conclusiones:

En cuanto a la categoría evaluación para la mejora continua, las docentes han identificado la existencia de algunas características propias de las escuelas eficaces, explicando en qué medida estas se dan en la institución. Al analizar estas características se las ha podido relacionar el impacto positivo/negativo que ha tenido -según las docentes entrevistadas- en el aprendizaje de los estudiantes y en su desempeño docente. En este punto, se identificó:

El objetivo evaluativo que tienen las docentes difiere, lo cual expone una desarticulación en los enfoques de enseñanza aprendizaje de cada una, dificultando el logro de objetivos de aprendizaje a nivel institucional.

El acompañamiento docente realizado por la institución es tomado como un elemento importante, puesto que invierten tiempo y dinero en ello, orientado hacia la mejora. Este factor promueve no solo mejora a nivel de clima institucional o sentimiento de soporte en los docentes, sino que sustenta un accionar institucional que facilita el aprendizaje.

La mejora continua a nivel institucional se da de manera poco articulada a modo de punto de partida para el inicio del siguiente año escolar -en el caso de la encuesta de fin de año- y de resolución de conflictos más que de autorreflexión institucional -en el caso de las reuniones bimestrales entre docentes y directivos-. A pesar de ello, el contar con espacios para el trabajo docente colaborativo, una comunicación constante, apertura institucional, buen trato dentro de la institución, respuestas inmediatas a situaciones problemáticas y capacitación previa a cambios son herramientas que facilitan la mejora continua y el bienestar docente. Es así que el orientar estos procesos se plantea como una necesidad fundamental.

Si bien la actualización docente se da con poca frecuencia, lo cual disminuye su impacto en la labor educativa, los docentes poseen un compromiso con su labor el cual las lleva a buscar de manera independiente oportunidades de aprendizaje autónomo.

El compromiso y la participación de los padres de familia en la formación de sus hijos es muy alto y juega un rol muy importante en el logro de los objetivos de aprendizaje.

Esta conclusión, describe de qué manera se dan el aprendizaje organizativo, el desarrollo profesional y el seguimiento y la evaluación en todos los niveles de la institución muestra (docente-estudiante, institución-docente e institución-institución). La existencia de los factores organizacionales previamente descritos ha sido identificada -en diferentes niveles de logro- por las docentes entrevistadas, permitiendo observar las fortalezas y las oportunidades de mejora existentes.

En cuanto a la categoría flexibilidad docente, los docentes han identificado la presencia de algunas características propias de las escuelas eficaces en la institución educativa a la cual pertenecen. Estas características demuestran un alto nivel de flexibilidad y adaptación por parte de las docentes, lo cual facilita las oportunidades de aprendizaje de los estudiantes:

Las docentes han manifestado tener en cuenta la existencia de diversidad de estilos de aprendizajes en sus aulas y contextualizar las actividades y la evaluación en base a esta diver- 
sidad, esto promueve un clima de aula inclusivo donde se promueve la sana convivencia, lo cual influye de manera positiva en el aprendizaje de los estudiantes.

El uso de recursos con propósito educativo ha sido mencionado también, pero este se centra más en la forma (necesidad de pizarras virtuales, proyectores, etc) que en la función que se le da (¿se sabe hacer uso de estos? ¿se empleará la tecnología, pero continuará con la misma metodología?).

Ambas conclusiones, toman como base los resultados obtenidos en la investigación y describen de qué manera se dan el aprendizaje organizativo, el desarrollo profesional, el seguimiento y la evaluación en todos los niveles de la institución muestra (docente-estudiante, institución-docente e institución-institución), la organización del aula, las expectativas que tiene el docente hacia el estudiante y la cultura escolar en la institución educativa. Asimismo, en el presente trabajo se hace evidente no solo la presencia de los tres factores organizacionales previamente mencionados, sino también se evidencia la presencia de otros como la calidad de la enseñanza y del currículo, el clima escolar y de aula, liderazgo educativo y pedagógico, sentido de comunidad, compromiso e implicación de la comunidad educativa y políticas escolares contextualizadas. La existencia de los factores organizacionales descritos ha sido detallada -en diferentes niveles de logro- por las docentes entrevistadas; permitiendo no sólo identificarlos sino también comprenderlos y contrastarlos, lo que nos ha permitido reconocer las fortalezas y las oportunidades de mejora existentes. Estas fortalezas y oportunidades le permiten a la institución replantear y mejorar el aprendizaje de sus estudiantes y crecer a nivel institucional; asimismo, brindan la oportunidad de originar futuras investigaciones. 
Báez, B. (1994). El movimiento de escuelas eficaces: Implicaciones para la innovación educativa. Revista Iberoamericana de Educación, (4). Recuperado de: https://rieoei.org/historico/ oeivirt/rieo4ao4.htm

Blanco, E. (2008). Factores escolares asociados a los aprendizajes en la Educación Primaria Mexicana: Un análisis multinivel. Revista Electrónica Iberoamericana sobre Calidad, Eficacia y Cambio en Educación, 6 (1), 58-82. Recuperado de: https://revistas.uam.es/index.php/reice/ article/viewFile/5454/5893

Blanco, E. (2009). Eficacia escolar y clima organizacional: apuntes para una investigación de procesos escolares. Revista Estudios Sociológicos, XXVII (80), 671-694. Recuperado de: http:// www.redalyc.org/articulo.oa?id=59820676011.

Blanco, R. et al (2008). Eficacia escolar y factores asociados en América Latina y El Caribe. UNESDOC, 12-58. Recuperado de: https://unesdoc.unesco.org/ark:/48223/pfooo0163174.

Carreras, P. (2013). Análisis de tres factores de un modelo de eficacia escolar aplicado por una organización privada en zona rural en el Perú. Recuperado de: http://tesis.pucp.edu. pe/repositorio/bitstream/handle/20.500.12404/4633/CARRERAS_MARIANI_PIERA_ ANALISIS_RURAL.pdf?sequence $=1 \&$ isAllowed $=y$

Cornejo, R.\&Redondo,J.(2007).Variables y factores asociados al aprendizajeescolar: Una discusión desde la investigación actual. Revista Estudios Pedagógicos, XXXIII (2), 155-175. Recuperado de: https://scielo.conicyt.cl/scielo.php?script=sci_arttext\&pid=S0718-07052007000200009

Cueto, S., Ramírez, C. \& León, J. (2003). Eficacia escolar en escuelas polidocentes completas de Lima y Ayacucho. Recuperado de: http://www.cies.org.pe/sites/default/files/investigaciones/ factores-de-eficacia-escolar-en-escuelas-polidocentes-completas-en-el-peru.pdf

Cuñat, R. (2007). Aplicación de la teoría fundamentada (Grounded Theory) al estudio del proceso de creación de empresas. XX Congreso anual de AEDEM, Decisiones basadas en el conocimiento y en el papel social de la empresa, Asociación Española de Dirección y Economía de la Empresa, (2), 1-13. Recuperado en https://dialnet.unirioja.es/descarga/articulo/2499458.pdf

Davis, G. \& Thomas, M. (1992). Escuelas eficaces y profesores eficientes. Madrid: La Muralla, 20150.

Díaz-Bravo, L. et al (2013). La entrevista, recurso flexible y dinámico. Investigación en Educación Médica, 2 (7), 162-167. Recuperado de: https://www.redalyc.org/pdf/3497/349733228009.pdf

Dörner, O. (2012). Experteninterviews. In B. Schäffer and O. Dörner. (Hrsg.). Handbuch Qualitative Erwachsenen und Weiterbildungsforschung. Opladen, Berlin \& Toronto: Verlag Barbara Budrich, 321-333.

Fernández, T. (2004). De las escuelas eficaces a las reformas educativas de segunda generación. Revista Estudios sociológicos, 22 (65), 377-408. Recuperado de: https://dialnet.unirioja.es/ servlet/articulo?codigo $=2192102$

Gamazo, A. et al (2018). Evaluación de factores relacionados con la eficacia escolar en PISA 2015. Un análisis multinivel. Revista de educación, (379), 56-78. Recuperado de: https://sede. educacion.gob.es/publiventa/evaluacion-de-factores-relacionados-con-la-eficacia-escolaren-pisa-2015-un-analisis-multinivel--assessment-of-factors-related-to-school-effectiveness-in-pisa-2015-a-multilevel-analysis/investigacion-educativa/22172

Granvik, M., Brolin, S., Almquist, Y. \& Modin, B. (2018). Effective schools, school segregation, and the link with school achievement. School Effectiveness and School Improvement, 464-484. Recuperado de: https://www.tandfonline.com/doi/full/10.1080/og243453.2018.1470988

Hofman, R., Hofman, W. \& Gray, J. (2015). Three conjectures about school effectiveness: An exploratory study. Cogent education, (2), 1-13. Recuperado de: https://doi. org/10.1080/2331186X.2015.1006977

Huamán, P. (2018). Valoración que otorgan los padres de familia de 6to grado de primaria del colegio Fe y Alegría $\mathrm{N}^{0} 1$ a los factores de eficacia escolar. 15-30. Recuperado de: http://tesis. pucp.edu.pe/repositorio/bitstream/handle/20.500.12404/14259/HUAMÁN_ROMERO_ PAMELA_NATY.pdf?sequence=1\&isAllowed $=y$ 
Instituto Internacional de Planeamiento de la Educación (2000). Gestión educativa estratégica: Diez módulos destinados a los responsables de los procesos de transformación educativa. 15-32. Recuperado de: https://educrea.cl/gestion-educativa-estrategica-diez-modulosdestinados-a-los-responsables-de-los-procesos-de-transformacion-educativa/

Murillo, J., et al (2000). La mejora de la eficacia escolar: Un estudio de casos. Recuperado de: https://oas.org/cotep/GetAttach.aspx?lang=en\&cld=392\&aid=613

Murillo, J. (2004). Un marco comprensivo de mejora de la eficacia escolar. Revista Mexicana de Investigación Educativa, 9 (21), 319-360. Recuperado de: http://www.redalyc.org/ pdf/140/14002104.pdf

Murillo, J. \& Martínez, C. (2016). Factores de eficacia escolar en la República Dominicana. Innovación educativa, 16 (72), 113-132. Recuperado de: http://www.scielo.org.mx/scielo. php?script=sci_arttext\&pid=S1665-26732016000300113

Murillo, J. \& Román, M. (2007). I Congreso Iberoamericano de Eficacia Escolar y Factores Asociados. REICE, 5 (5). Recuperado de https://revistas.uam.es/index.php/reice/article/ view/10112

Murillo, J. (2008). Hacia un modelo de eficacia escolar. Estudio multinivel sobre los factores de eficacia en las escuelas españolas. Revista Electrónica Iberoamericana sobre Calidad, Eficacia y Cambio en Educación, 6 (1), 4-28. Recuperado de: http://www.redalyc.org/pdf/551/55160102. pdf

Ruiz, F. \& Parrilli, M. (2015). Sobre flexibilidad educativa y el rol docente. Revista de Informática Educativa y Medios Audiovisuales, 12 (19), 1-4. Recuperado de: http://laboratorios.fi.uba.ar/ lie/Revista/Articulos/121219/A1.pdf

San Martin, D. (2014). Teoría fundamentada y Atlas.ti: recursos metodológicos para la investigación educativa. Revista Electrónica de Investigación Educativa, 16(1), 104-122. Recuperado de http://redie.uabc.mx/vol16no1/contenido-sanmartin.html

Secretaría de Educación Pública (2010). Modelo de Gestión Educativa Estratégica. Recuperado de: http://formacion.sigeyucatan.gob.mx/formacion/materiales/5/d3/p3/3.\%20EL\%20 MODELO\%20DE\%20GESTION\%20EDUCATIVA\%20ESTRATEGICA.pdf

Stoll, L. \& Dean, F. (1999). Para cambiar nuestras escuelas. Reunir la eficacia y la mejora. Madrid: Octaedro.

Taylor, S. \& Bogdan, R. (1984). La observación participante en el campo. Introducción a los métodos cualitativos de investigación. La búsqueda de significados. Barcelona: Paidós Ibérica. 